A puntes del CENES

ISSN 0120-3053

Volumen 30 - №. 52

Segundo Semestre 2011

Págs. 103 - 116

\title{
Incidencia de poderes exógenos en las políticas públicas y en el sector rural en Colombia
}

\section{Incidence of exogenous powers in public policies and in the rural sector in Colombia}

Óscar Cardozo Londoño*

Fecha de recepción: 28 de marzo de 2011

F echa de aprobación: 30 mayo de 2011

Economista, especialista en Proyectos de Desarrollo, magíster en Desarrollo Rural, catedrático Facultad de Estudios a Distancia, Universidad Pedagógica y Tecnológica de Colombia. Correo electrónico: oscar.cardozo@uptc.edu.co. El autor agradece los conceptos y sugerencias de los pares anónimos que ayudaron a mejorar el documento. 
Incidencia de poderes exógenos en las políticas públicas y en el sector rural ...

Ó scar Cardozo Londoño

\section{Resumen}

El presenteescrito analiza de quémanera, a lo largo dela historia colombiana, una serie de fuerzas y poderes ajenos al sector rural, han ejercido una influencia nociva para este. Se estudia desde diver sas per spectivas, el impacto que esas fuerzas y poderes han tenido en detrimento de uno de los sectores más importantes para el desarrollo del país, teniendo en cuenta que el sector rural ha sido predominante en Colombia. Por último se hace un acercamiento al debate actual sobre políticas públicas agrarias desde diferentes miradas, con el fin de contribuir al estudio y comprensión del caso colombiano.

Palabras clave: cuestión agraria, políticas públicas, política rural, política agraria, estructuras productivas, territorio, conflicto.

Clasificación JEl: P11, P15, P18, P25, P28, Z18, J18, F51

\section{Abstract}

This paper analyzes how, throughout Colombian history, a series of forces and powers foreign to the rural sector, have exerted a harmful influence for it. It is studied from different perspectives the impact that these forces and powers have had in detriment to one of the most important sectors for the development of the country, bearing in mind that the rural sector had been predominant in Colombia. Finally, it is made an approach to the current debate on agricultural public policies, from various views, in order to contribute to the study and understanding of the Colombian case.

Keywords: agrarian question, public policies, rural policy, agricultural policy, productive structures, land, conflict.

Jel Clasification: P11, P15, P18, P25, P28, Z18, J18, F51 


\section{Introducción}

El presente escrito analiza como a lo largo de la historia colombiana, una serie de fuerzas y poderes ajenos al sector rural han ejercido una influencia nociva para este. En el documento se estudia desde diversas perspectivas el impacto que esas fuerzas y poderes han tenido en detrimento de uno de los sectores predominantes y más importantes para el desarrollo del país.

Para el presente documento se acogen mayormente las hipótesis planteadas por Fabio Lozano (2010), en el sentido de que "Existe un enorme contraste entre los sucesos acaecidos por la fuerza de los poderes militares, económicos y políticos y las formulaciones de política pública, que aparentando un interés de equidad y solución de necesidades sociales, en realidad constituye un sistemático y permanente tejido de legitimación de usurpaciones y de situaciones de dominación". Además, en este documento se abordan otras aproximaciones, para indicar que usualmente desde el Estado no se toman políticas agrarias dentro de un marco de políticas públicas, sino que se toman decisiones de política agraria de corte puramente coyuntural (Machado, 2002). Estas políticas ayudan a consolidar poderes internos (Lozano, 2010) y externos -no solo al sector rural sino a la nación misma-, al permitir el empoderamiento de las multinacionales y transnacionales.

El análisis propuesto por Lozano (2010) indica que las políticas públicas deberían ayudar a la solución de problemas que se generan en el sector agrario, sin embargo, en realidad, han cumplido el papel de ayudar a la consolidación de 
poderes internos y externos sobre las poblaciones rurales. Partimos del ejemplo concreto de la asignación del subsidio denominado Agro Ingreso Seguro en el Gobierno del expresidente Álvaro Uribe Vélez y casos como el de Carimagua donde se asignó el subsidio en primer lugar y luego se pretendió asignar grandes recursos del Estado a poderosos grupos, en detrimento de los más necesitados, como los desplazados, los campesinos y los pequeños productores.

En este escrito se hace un recorrido por la historia colombiana desde la época de la Conquista y la Colonia hasta las dos últimas décadas del siglo XX, para continuar con los enfoques de política agraria estatal y terminar con algunas propuestas, que, más que eso, son interrogantes sobre la posición que se debería adoptar hacia el futuro. De la misma manera, se revisan distintas posiciones de varios autores sobre el tema; luego se sustenta una posición y finalmente se presentan algunas conclusiones.

\section{Antecedentes de la política agraria en Colombia}

El primer choque se presentó en el territorio de los Zenues, cuando un tal Alonso de Enciso le notificó a un cacique que las tierras que habitaba no eran suyas sino del rey, quien las había recibido del papa. Airado, el cacique le respondió que si el papa había regalado esas tierras era porque debía haber estado borracho y si el rey las había recibido de quien no era su dueño era porque debía estar loco, y añadió que viniera el rey a tomar esas tierras si era capaz. Ese fue el inicio de la reacción agraria en Colombia (Mendoza, 2007).

Se continuó con el modelo hacendatario, que forjó la ética particular de cada una de las etnias que forman nuestra nacionalidad: el blanco: "cómo me quedo con todo", dando origen a la aún presente "terrofagia"; el indígena: "cómo me defiendo de la agresión", dando origen a la llamada "malicia indígena"; el negro: "cómo me libero", dando origen a las sublevaciones, a los palenques y al concepto de "territorios libres" (Mendoza, 2007).

Posteriormente vienen reformas encaminadas a contrarrestar la ambición, la codicia y la agresividad de los colonizadores, tales como la reforma de 1591 de Felipe II, que reclama respeto para los bienes reales por parte de los mismos españoles (Mendoza, 2007).

Durante la Colonia, los jesuitas crearon el modelo de las "reducciones", modelo progresista para su época, por lo cual fueron expropiados en 1767 y expulsados de América, sus tierras fueron entregadas a grandes latifundistas, como un funesto antecedente de la situación actual. En 1785, el visitador Antonio Mon y Velarde implantó una reforma agraria con la que expropió tierras y las repartió; de esta 
época también se destaca la creación de escuelas agrarias.

En la época de la Independencia, los "libertadores" disolvieron los resguardos, que eran la única institución protectora de los indígenas. Posteriormente Simón Bolívar los restableció en 1820, y ordenó devolver las tierras a los indígenas como propietarios legítimos de las mismas. En 1850 se disuelven nuevamente los resguardos mediante una ley del 22 de junio, que dispuso su libre enajenación -que se prolongó hasta 1931-, fecha a partir de la cual se han restablecido antiguos resguardos y creado algunos nuevos.

Más adelante, en 1861, Tomás Cipriano de Mosquera decretó la expropiación de bienes de la Iglesia y la desamortización de los bienes llamados de "manos muertas" (Tirado, 1988). Salvador Camacho Roldán dijo que era "la reforma social de más fecundos resultados desde la supresión de la esclavitud y los mayorazgos" (Mendoza, 2007). Pero finalmente las tierras fueron compradas por terratenientes, especuladores y políticos que terminaron sumándose al régimen tradicional de latifundio como había sucedido en el caso de los jesuitas.

Alfonso López Pumarejo promulga en 1936 la Ley 200, que contenía el principio de la función social de la tierra, reforma que fracasó dada la cerrada oposición parlamentaria, con amenazas de guerra civil en caso de aplicarse. Algunos estudiosos del tema, como
Viviana Vargas (2007), consideran que la Ley 200 es "el primer intento serio de realizar una reforma agraria y fue el resultado de una concertación con el movimiento campesino en auge. Elementos importantes de la ley eran la creación de jueces especiales para dirimir conflictos de tierra, la introducción del concepto de la función social de la tierra y la figura de extinción de dominio". A pesar de su reducida aplicación por la fuerte resistencia que encontró, la Ley 200 erigió las bases conceptuales de la reforma agraria.

Según Gabriel Tobón, la Ley 100 de 1944 significó un retroceso con respecto a los avances alcanzados en la Ley 200 de 1936, ya que se orientó a neutralizar sus efectos al declarar de conveniencia pública el incremento de los cultivos mediante los sistemas de arrendamiento y aparcería, con lo cual se garantizó la sujeción de los campesinos sin tierra y de su mano de obra al poder de los terratenientes, evitando su conversión a campesinos con tierra; además permitió a los propietarios dinamizar sus rentas frente a estos sistemas de explotación y provocó su ausentismo (Tobón, 1998).

En 1950, Lauchin Currie -el mismo ideólogo del UPAC-, propuso en la "Operación Colombia" una política "anticampesinista y prourbana", en el sentido de traer los campesinos a las ciudades (Lozano, 2010b). Se necesitaba una masa de trabajadores que impulsaran a los sectores que hacían desarrollar la economía haciéndola más competitiva 
como la industria, las finanzas y los servicios; su propuesta fue aceptada contribuyó a la despoblación del campo, la congestión de las ciudades y el encarecimiento de la vivienda.

Entre 1950 y 1960, se presentó una ola de reformas agrarias en distintos países en todo el mundo, con su consecuente impacto en Latinoamérica y en Colombia. A raíz de casos como la Revolución Cubana y de la sublevación de grandes masas de campesinos sin tierra, surge el interés de Estados Unidos y de los organismos internacionales de dar financiación a nuestros países con el fin de evitar un desequilibrio en la balanza política a favor de la Unión Soviética. Estos hechos tuvieron una notable influencia en Colombia donde los diferentes actores sociales, políticos, intelectuales, académicos e institucionales se nutrieron de esas teorías, las cuales se empezaron a expresar en el debate agrario (Tobón, 1998, p. 30-31).

Carlos Lleras Restrepo impulsó en 1968 una reforma agraria fundada en empresas comunitarias, que al principio prosperó, pero posteriormente la mayoría desapareció y quedan muy pocas en estado de decaimiento. En 1972, un grupo de propietarios reunidos en Chicoral, (Tolima), suscribió un acuerdo conocido como el "Pacto de Chicoral", o "Chicoralazo". Este acuerdo es un modelo de contrarreforma agraria apoyado en la gran propiedad como fundamento de desarrollo agrario, que dirige el crédito hacia los empresarios agrícolas, desmonta la reforma agraria y se pierde el apoyo alcanzado. La reforma agraria fue terminada y en su lugar se establecieron programas de asistencia técnica que se dirigían a los minifundistas.

Entre 1961 y 1972 se dieron dos hechos importantes. Primero, la organización institucional con el fin de manejar de mejor manera la reforma agraria mediante la creación del Instituto Colombiano de la Reforma Agraria INCORA (Ley 135 de 1961), que buscaba obligar a los terratenientes a modernizar sus explotaciones y a darle un uso adecuado a la tierra. En segundo lugar, se presenta la mayor acción reformista desde el Estado. La legislación que sufre profundas modificaciones en el Congreso es resultado de múltiples interacciones y presiones ejercidas por el movimiento social agrario (Tobón, 1998, p. 33-37). Pero como la dirección del proceso de reforma agraria estaba muy politizada y contaba con escasa participación campesina, finalmente los más beneficiados fueron los terratenientes, en especial en el Sumapaz y en el sur del Tolima.

En 1967 se constituyó la Asociación Nacional de Usuarios Campesinos (ANUC), utilizada por el presidente Lleras como factor de presión para llevar a cabo su reforma agraria, que tomó forma con la Ley $1^{a}$ de 1968 , que dio impulso a la organización y participación campesina, facilitó los trámites, hizo énfasis en los predios inadecuadamente 
explotados y en la entrega de tierra a los aparceros que la trabajaban.

La Ley 4 de 1973 estableció criterios para la clasificación de los predios con el objetivo de expropiación, pero designaba casi cualquier predio como adecuadamente explotado, y sus trámites diluyeron la posibilidad de hacer una reforma agraria. La Ley 6 de 1975 legalizó de nuevo la aparcería, pero a pesar de que buscaba la modernización de las relaciones entre propietario y aparcero, el principio de sujeción del trabajador se mantuvo. En ese año se impuso el Programa de Desarrollo Rural Integrado (DRI), que no fue concebido como un complemento de la reforma agraria, sino como su sustituto y que resultó ser insuficiente y no logró cambiar de manera sustancial la situación del campesinado. Con la Ley 35 de 1982 y el Plan Nacional de Rehabilitación se retomó la cuestión agraria, pero bajo otro esquema: la compra de tierra a precios de mercado y su venta a campesinos beneficiarios.

En 1988 se aprobó la Ley 30 que formaliza la compra de tierra a precios de mercado. De esta manera se quería vencer la resistencia de los terratenientes, y las adquisiciones del Incora alcanzaron niveles muy altos. Este procedimiento de reforma agraria es limitado por restricciones presupuestales, de modo que la reforma siguió siendo marginal (Vargas, 2007).

Vargas (2007) cita a Antonio García, como así la reforma agraria en Colombia ha tenido carácter marginal: se ha tratado de una reforma dirigida a mantener el statu quo del complejo latifundio o minifundio, con concesiones a la mediana propiedad, a través de la canalización de las presiones sobre la tierra hacia las fronteras agrarias. Además, se ha privilegiado la titulación de baldíos y afectado las tierras del interior solamente en forma ligera, cuando lo ha exigido la confrontación social.

Entre 1973 y 1998, se implementan los Programas de Desarrollo Rural Integrado y de Alimentación Nacional PAN, que van a desplazar y subordinar los programas de reforma agraria como estrategias de desarrollo rural, y, en su lugar, marcan un nuevo énfasis y orientación de los organismos de cooperación y la banca internacionales. También se presentan otros fenómenos como la movilización social campesina, el desmonte y los sustitutos de la reforma agraria, los nuevos procesos de contrarreforma agraria, la nueva orientación de las políticas de Estado: "El mercado" (Lozano, 2010). La fragmentación del movimiento campesino y el desplazamiento masivo y forzoso de la población rural -grandes latifundios en la Costa Atlántica, en especial en los departamentos de Sucre, Córdoba y Bolívar-, acentuado en los últimos treinta años con el pretexto del conflicto armado y con la incidencia del fenómeno del narcotráfico, igualmente en algunos casos se permitió la dominación 
paramilitar de la población so pretexto de dar seguridad a los grandes inversionistas.

En el período descrito, el problema agrario colombiano se ha agravado por la concentración de la tierra y el poder en pocas manos, el uso ineficiente de la explotación y producción de la tierra, al cambiar su vocación agrícola y forestal por la de ganadería extensiva.

De acuerdo con el planteamiento inicial sobre la injerencia de poderes y fuerzas externas a la nación, y en especial al sector rural, puede verse que en 1990 a partir del llamado "Consenso de Washington", se formuló una serie de políticas consideradas por los organismos financieros internacionales como el programa económico que debían aplicar los países latinoamericanos. Estas incluían el reordenamiento de las prioridades del gasto público, la liberalización del comercio internacional, el permiso de la entrada de inversiones extranjeras directas, la privatización, la desregulación y reformas impositivas. Este "consenso" se vio más como una forma de abrir los mercados latinoamericanos para ser explotados por los países desarrollados. Por otra parte, puede verse que estas políticas se imponen a países débiles, y que no han producido ningún aporte significativo, en cambio sí han ocasionado distorsiones y crisis, por ejemplo, el aumento en la deuda externa, lo cual mantiene a nuestros países en condiciones de pobreza y subdesarrollo.

\section{Interpretaciones de las políticas agrarias}

A partir de este marco histórico es importante revisar las categorías de interpretación y el marco de los enfoques de políticas públicas que se han implementado, (teniendo en cuenta el precepto según el cual el objetivo fundamental de cualquier política pública (educación, salud, etc.), es garantizar el bienestar de la población (Lozano, 2010). Con este fin se utilizará la guía de enfoques de política agraria propuesta por Fabio Lozano (2010).

Las políticas agrícolas en el mundo se pueden clasificar en cinco grandes grupos (Machado, 2002): el primero corresponde a los países que no tienen una política agrícola, según su propia opinión, como Argentina, Australia y Nueva Zelanda. Estas naciones pertenecen al Grupo Cairns, en el que prevalece la política macroeconómica y no se aboga por políticas sectoriales. El segundo grupo lo componen Estados Unidos y la Unión Europea, con políticas a largo plazo para la agricultura, proteccionismo e importantes ayudas para este sector. El tercer grupo lo constituyen países que tienen leyes con orientación hacia la agricultura, como Francia. El cuarto grupo lo conforman países con leyes rectoras para la agricultura como Japón y Corea, que no reglamentan aspectos específicos pero le dan un norte a la política. El quinto grupo está conformado por países en vía de desarrollo, donde se hacen intentos de aplicar políticas agrícolas de corte 
coyuntural, buscando una política activa que tiene grandes contradicciones con las políticas macroeconómicas. Son países donde no se ha podido llegar aún a una visión a largo plazo del Estado sobre la agricultura, como Colombia, los países del Grupo Andino, de Centroamérica y de África.

Este último grupo de países está en la órbita del tercer mundo (Machado, 2002, p. 152-153). Se caracteriza por tener Estados débiles, con bajos recursos para apoyar la agricultura, sometidos a políticas trazadas por organismos internacionales, fuertemente endeudados, que no alcanzan ni a pagar los intereses de la deuda, con ministerios de agricultura sin capacidad de respuesta a las demandas de los agricultores y productores rurales, que no tienen políticas agrícolas a largo plazo, con legislaciones caóticas y poco coherentes. Son países con procesos de apertura económica, lo cual los obliga a manejar políticas macroeconómicas y a descuidar las políticas sectoriales, lo que genera abandono al sector agropecuario.

Estos países están muy influidos por las políticas agrícolas de los países más industrializados, en la medida en que sus productos de exportación buscan acceso a sus mercados y que los subsidios que se otorgan en los industrializados, crean una competencia desleal difícil de enfrentar. Jesús Antonio Bejarano, -citado por Machado (2002)- da las pautas para la construcción de una verdadera política agrícola: la necesidad de una política sectorial activa, definir una política de transición en el marco de una nueva política comercial, redimensionar el papel del desarrollo rural, fortalecer la capacidad institucional y operacional respecto de los nuevos temas de la agenda y mejorar la gobernabilidad de las instituciones políticas públicas.

Los neoliberales desean crear un marco económico que sea aplicable a todos los sectores sin hacer distinciones entre agricultura, industria y servicios. Se oponen a las políticas sectoriales porque creen en el desarrollo de un escenario macroeconómico estable y uniforme (Kay, 2010, p. 39-42), en concordancia con lo expuesto por Machado. Kay (2010) presenta los cinco paradigmas de desarrollo rural que se han aplicado en América Latina en los últimos sesenta años: modernización, estructuralismo, dependencia, neoliberalismo y neoestructuralismo.

El mismo autor dice que las políticas de los países latinoamericanos se han visto cada vez más afectadas por el paradigma del neoliberalismo dominante en la actualidad, que se centra en cinco áreas principales: gestión fiscal, privatización, mercado de trabajo, comercio y mercados financieros. Además reitera el argumento de fondo según el cual en la medida en que los gobiernos se comprometen con políticas neoliberales tienen que aproximarse a la gestión macroeconómica, haciendo énfasis en el gasto público que afecta a los sectores más sensibles como el sector rural. 
Kay (2010) indica en su análisis que en las últimas décadas se han presentado contribuciones importantes desde distintos campos del desarrollo rural sobre temas como desarrollo sostenible, capital social y nueva ruralidad, y que todo debe analizarse en conjunto dentro de una problemática más amplia y no de una forma aislada en los procesos de desarrollo, a fin de que evolucionen hasta configurar un paradigma propio. Kay (2010) lamenta que el paradigma dominante sea el neoliberalismo y no un paradigma de desarrollo rural construido sistemáticamente que supere los marcos teóricos actuales.

Pedro Medellín (2004) propone un modelo teórico y metodológico para el estudio de políticas públicas en países con frágil desarrollo político e institucional, a partir de la hipótesis según la cual la naturaleza del régimen político determina la estructuración de políticas públicas. Hay que tener en cuenta que en países como Venezuela, Bolivia, Perú o Colombia no hay una planeación institucional de políticas públicas, los sistemas de control son rígidos, existe excesiva concentración del poder, e inestabilidad de los funcionarios en cargos de decisión con sus cambios de agenda, todo esto en el marco de una informalidad institucional y de una precariedad política (Medellín, 2004, p. 7). Según Medellín, las políticas públicas solo pueden ser entendidas en el marco del régimen político del cual emergen, no son variables independientes que puedan ser explicadas de manera ajena al conjunto de factores institucionales y políticos en el cual se estructuran (2004, p. 8). Presenta su sustentación en cuatro partes, dentro de las cuales puede resaltarse la segunda, en la cual se asume que el grado de consolidación política e institucional es el criterio clave para establecer las diferencias entre regímenes políticos, y que dentro de estos, los márgenes de autonomía son el factor determinante para establecer políticas públicas.

En la última parte de su documento, Medellín analiza el modo como se estructuran las políticas públicas para regímenes caracterizados por un frágil desarrollo político e institucional. Las políticas públicas son el medio por el cual el gobierno puede poner en marcha la maquinaria gubernativa, y la función gubernativa tiene un carácter integrador en las distintas esferas del Estado para lograr un fin específico, tal como aunar las fuerzas de la sociedad en torno a un determinado proyecto $(2004$, p. 16$)$.

Concluye Medellín (2004), que los procesos de estructuración de políticas públicas están sometidos a complejos procesos de negociación de intereses externos e internos a los gobiernos, en un escenario conflictivo y fragmentado, marcado por los compromisos electorales, donde cada quien pelea por sus intereses y beneficios. En ese escenario, la toma de decisiones está determinada por la magnitud e intensidad de las presiones internas y externas al gobierno -como ya se anotó- y la 
elaboración de la agenda operacional sectorial queda supeditada a los compromisos adquiridos por los funcionarios de alto nivel.

La estructuración de las políticas públicas está determinada por la manera particular como los gobernantes disponen los recursos institucionales y las prácticas culturales de gobierno para obtener los resultados deseados; por la lucha entre las distintas fuerzas de poder por imponer desde el gobierno (o contra él) un determinado proyecto de dirección política y un proyecto de dirección ideológica al Estado y a la sociedad; por el grado en que la distribución del poder estatal se distorsiona en poder burocrático que emerge para interferir (o potenciar) la consecución de los objetivos de gobierno; por el tipo de instancias y mecanismos comunicacionales que rigen las relaciones entre gobernante y gobernados; y por el grado en que las relaciones intergubernamentales se constituyen o no en una correa de transmisión de las acciones y decisiones de gobernantes y gobernados en el logro de un proyecto de gobierno (Medellín, 2004, p. 43-45).

En este complejo juego de intereses, en el que la multiplicidad de fines y medios, así como el conjunto de restricciones y factores de bloqueo institucional limitan la capacidad de acción gubernamental, en condiciones de una institucionalidad frágil, la estructuración de políticas públicas queda determinada por la diseminación de poderes y por la primacía de los intereses privados sobre el interés público.

Para Viviana Vargas (2007), el acceso a la tierra en Colombia ha sido históricamente una gran fuente de poder político y de conflicto social, además un determinante clave en la productividad de la economía. "La expansión del conflicto se ha profundizado en torno al control de territorios estratégicos por parte de grupos paramilitares y esto ha generado desplazamiento de comunidades enteras". Indica que para los campesinos hablar de una reforma agraria significa terminar con una estructura que a lo largo de la historia ha subordinado sus vidas, cultura e intereses, para decidir por primera vez como sujetos sobre su destino como comunidades campesinas.

Entre las posiciones en contra, se encuentra la de Alfonso López Caballero (1987), posición neoliberal en la cual se concibe la problemática agraria desde una visión productivista (la competitividad) de los mercados y la disminución de la acción del Estado. Su discusión no se centra en la estructura agraria, sino en el desarrollo de los mercados de factores, incluida la tierra, y en los incentivos para dinamizarlos en vez de redistribuirlos. Según este enfoque, si la concentración de la propiedad rural se articula con la productividad, el Estado no debe intervenir, en las economías campesinas sino que estas deben modernizarse. El 
Estado solo debe mantener temporalmente programas de desarrollo rural. López Caballero señalaba a principios de la década de los ochenta que el problema no era únicamente de redistribución de tierras, sino de calidad de vida (pobreza) y de capacidad productiva reprimida por las distorsiones estructurales del modo de producción agrícola.

Para López (1987), la gran propiedad es ineficiente en el uso de la mano de obra, por lo tanto debe recomponerse el tamaño de la propiedad para potenciar la producción. Una agricultura de pequeñas y medianas fincas con acceso a capital y tecnología es lo requerido para aumentar el nivel de ingreso y bienestar. Según Absalón Machado (2002), ese es un argumento economicista que se limita a circunscribir la redefinición del tamaño de la propiedad sin tener en cuenta las relaciones sociales, los poderes políticos, la injerencia de los terratenientes en el Estado y las condiciones de atraso de la sociedad rural.

A partir del oscuro panorama que se ha presentado a lo largo de la historia para el sector rural, la primera pregunta que surge es: ¿qué hacer desde lo local? Para responder esta pregunta se debe partir del supuesto que nada se logra únicamente a partir de buenas intenciones, que la solución no está "a la vuelta de la esquina" ni mucho menos en los favores que haga el Banco Mundial, el Fondo Monetario Internacional o el Banco Interamericano de Desarrollo; tampoco se trata de tomar como "vox Dei" las fórmulas del "Consenso de Washington"; que debemos presentar propuestas concretas con alternativas, por ejemplo: ¿cómo fortalecer a los grupos vulnerables? "Al hacer política agraria se debe tener en cuenta que lo que está en juego no es la solución de una necesidad básica inmediata sino el dominio del territorio" (Lozano, 2010).

\section{Conclusiones}

El análisis efectuado permite ver que el Estado ha sido incapaz de resolver el problema de la tenencia de la tierra en Colombia, y de articular los planes de reforma agraria a los planes de desarrollo en sus diferentes niveles (nacional, departamental, municipal), permitiendo que la propiedad se concentre cada vez más en reducidos grupos de terratenientes, manteniendo un estado de inequidad social en el campo, que se traduce en pobreza y marginamiento, que hace que los conflictos sociales, políticos, económicos, culturales y ambientales se agudicen, afectando a los grupos más desprotegidos, lo cual, de continuar así, puede hacer inviable el Estado social de derecho.

Puede concluirse que cualquier propuesta de reforma agraria debe estar relacionada con los problemas más sensibles que afectan al sector rural: superar la pobreza, construir la paz, garantizar la seguridad y, sobre todo, la soberanía alimentaria para todos los colombianos, tener una democracia incluyente que permita el libre ejercicio de una plena soberanía. 
Estos temas deben ser prioritarios, no solo en la agenda, sino en la mente de los sectores políticos, gremiales, sociales, gubernamentales. También deben estar en la agenda de los investigadores sociales, para plantear propuestas creativas a la luz de las nuevas realidades, cambios y transformaciones, y del entorno de globalización.

Igualmente, deben diseñarse políticas públicas que estén más allá de un enfoque tecnocrático, políticas en las cuales intervengan todos los actores, -políticos, profesionales, investigadores, miembros de la sociedad civil-, que sean resultado de transacciones intemporales entre ellos, que tengan como características, estabilidad, adaptabilidad, coordinación y coherencia, calidad en su implementación, aplicación efectiva, y, sobre todo, eficiencia y orientación al interés público, como lo menciona Stein (2006).

Es importante promover políticas efectivas en contra del desplazamiento, como lo señalan Deininger y Lavendez (2004): las políticas para prevenir el desplazamiento son una prioridad urgente, se necesita ejecutar cinco cosas claves: la primera, reorientar tres factores estructurales que son base del desplazamiento: desigualdad, bajo gasto público, inseguridad. En segundo lugar, fortalecer a las poblaciones locales para resistir con el fin de disminuir las posibilidades de que sean desplazadas, o al menos mejorar sus oportunidades de regresar. En tercer lugar, proveer pronta asistencia a las personas desplazadas. En cuarto lugar, ayudarlas a retornar, a integrarse: algunos grupos como los hogares encabezados por viudas son menos propensos a regresar; y en quinto lugar, facilitar el retorno a donde ellos lo deseen, hay que tener en cuenta que los poseedores de tierras o quienes las cultiven tienen una alta posibilidad de retornar. Es importante también mejorar su seguridad física, asistirlos y asegurarles sus derechos.

Por último, las políticas agrarias no pueden universalizarse, deben analizarse las diversidades, deben reconocerse las experiencias de las organizaciones sociales que han tenido resultados positivos, aunque sean parciales, en cada país, por ejemplo, el caso del movimiento de los trabajadores sin tierra de Brasil ${ }^{1}$. Las nuevas políticas agrarias deben tener en cuenta elementos emergentes que no se han integrado o se han dispersado, tales como: política de aguas frente a política de bosques. Las políticas públicas no siempre son formuladas desde el Estado, las comunidades construyen procesos alternativos, y desde la academia también deben hacerse aportes críticos.

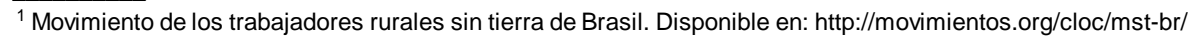




\section{Referencias bibliográficas}

1. Bejarano, J. A. (1998). Economía de la agricultura. Bogotá: TM Editores, Universidad Nacional de Colombia e IICA.

2. Deininger, K. \&Lavéndez, I. (Octubre, 2004).Colombia: landpolicy in transition. s.l.: a. Banco Mundial.

3. Lozano, F. \&Osorio, F.(1996). Población rural desplazada por violencia en Colombia.En Cuadernos de desarrollo rural. Vol. 36. Bogotá: Javegraf,p. 7-26.

4. Lozano, F. (Abril, 2010). De la reforma agraria al dominio territorial. Conferencia magistral llevada a cabo en la Maestría de Desarrollo Rural,Modulo de política agraria, Universidad Pedagógica y Tecnológica de Tunja.

5. Mendoza, A. (s.f.). Tesis agraria para Colombia. Recuperado en mayo de 2011 dehttp://www.sogeocol.edu.co

6. Kay, C. (2010). Los paradigmas del desarrollo rural en América Latina. La Haya:Institute of Social Studies.

7. Machado, A. (2002). De la estructura agraria al sistema agroindustrial. Bogotá: Universidad Nacional de Colombia.
8. Medellín, P. (2004). La política de las políticas públicas: propuesta teórica y metodológica para el estudio de las políticas públicas en países de frágil institucionalidad. Políticas sociales (93)CEPAL.

9. Movimiento de los trabajadores rurales sin tierra de Brasil. (s.f.). Recuperado junio 2011 de http://movimientos.org/cloc/mstbr/

10. Stein, P. (2006).Informe Banco Interamericano de Desarrollo.s.1.: Fedesarrollo.

11. Tirado, A.(1988).Introducción a la historia económica de Colombia. Bogotá: El Áncora.

12. Tobón, G.(1998).La reforma agraria una mirada desde lo público. Tesis de Maestría. Universidad de los Andes, Bogotá.

13. Tobón, G. (2007).Lo público, políticas públicas y políticas agrarias. En Colección Apuntes de clase. Bogotá:Pontificia Universidad Javeriana.

14. Vargas, V. (s.f.). La tenencia de la tierra en Colombia.Recuperado en enero de 2012, de http://www.prensarural.org/spip/ spip.php?article1288 